nervous system. Shalet et $a l^{2}$ in Manchester investigated 15 children who had received prophylactic craniospinal radiotherapy; most showed a decrease in linear growth and four had growth hormone deficiency. The reduction in the growth hormone response was greater in children who had had the radiotherapy in 10 fractions than in those who had received 20 fractions. The Manchester group ${ }^{3}$ has also found evidence of abnormalities in thyroid-stimulating hormone (TSH) and corticotrophin (ACTH) in some children. As yet it is not clear whether these abnormalities (which appear to be due to radiation damage to the hypothalamic-pituitary region) are common, permanent, and clinically important.

These children seem to have a good chance of normal development at puberty. A retrospective analysis of girls treated for leukaemia showed that $80 \%$ overall (more for those diagnosed before puberty) had normal pubertal progression and reproductive function. ${ }^{4}$ Most boys, too, seem to progress through puberty normally, but little is known about the influence of chemotherapy on spermatogenesis. Cyclophosphamide, which is known to cause azoospermia, ${ }^{5}$ is not a component of most current treatment regimens. The recent increase in incidence of testicular infiltration, ${ }^{6}$ however, suggests that prophylactic testicular irradiation may be desirable-which would inevitably cause sterility.

There are as yet only isolated reports of children born to survivors of acute leukaemia, and the incidence of defects in the offspring of survivors is not known. But a study of the progeny of survivors of childhood cancer ${ }^{7}$ (including, inevitably, few with leukaemia) showed no excess of children with birth defects.

Methotrexate, the drug most widely used in long-term chemotherapy, is toxic to the lungs, skeleton, and liver. ${ }^{8}$ Portal fibrosis and cirrhosis have been described both in patients treated for psoriasis ${ }^{9}$ and in patients with leukaemia. Methotrexate hepatotoxicity may be associated with minimal abnormalities in conventional liver function tests, and its extent can probably be accurately assessed only by liver biopsy. ${ }^{10}$

Methotrexate is also toxic to the central nervous system, especially in patients who have received cranial irradiation. Progressive neurological deterioration associated with disseminated necrotising leucoencephalopathy was first described in patients treated with long-term systemic and intrathecal methotrexate $^{11}$ for overt leukaemic infiltration of the central nervous system. Methotrexate-radiation encephalopathy has also occurred, however, in patients who had received prophylactic cranial irradiation and had later been given parenteral methotrexate in moderate dosage. ${ }^{12} 13$ The incidence of more subtle neuropsychological impairment remains uncertain. A preliminary neuropsychological evaluation of children treated for leukaemia ${ }^{14}$ showed no differences between patients and controls. But another small retrospective study showed that, while all children who had completed treatment were functioning within the normal range, younger children performed less well than their controls, especially in tasks requiring memory and motor skills. ${ }^{15}$

These preliminary reports all serve to emphasise the need for careful prospective studies aimed at minimising the late side effects of treatment. The extent of other problems (such as the risk of second neoplasms, apparent in patients with Hodgkin's disease ${ }^{\mathbf{1 6}}$ ) may not emerge for several years. Longterm surveillance of these children is clearly essential-but not, it is to be hoped, at the expense of making them feel perpetual patients.

${ }^{1}$ Aur, R J A, et al, New England fournal of Medicine, 1974, 291, 1230.
2 Shalet, S M, et al, Archives of Disease in Childhood, 1976, 51, 489.

${ }^{3}$ Shalet, S M, et al, fournal of Pediatrics, 1977, 90, 920.

4 Siris, E S, Leventhal, B G, and Vaitukaitis, J L, New England fournal of Medicine, 1976, 294, 1143.

${ }^{5}$ Fairley, K F, Barrie, J V, and Johnson, W, Lancet, 1972, 1, 568.

${ }^{6}$ Eden, O B, et al, British Medical fournal, 1978, 1. In press.

${ }^{7} \mathrm{Li}, \mathrm{F} \mathrm{P}$, and Jaffe, N, Lancet, 1974, 2, 707.

${ }^{8}$ Nesbit, M, et al, Cancer, 1976, 37, 1048.

${ }^{9}$ Dahl, M G C, Gregory, M M, and Scheuer, P J, British Medical Fournal, $1971,1,625$.

${ }^{10}$ McIntosh, S, et al, fournal of Pediatrics, 1977, 90, 1019

${ }_{11} \mathrm{Kay}, \mathrm{H} \mathrm{E} \mathrm{M}$, et al, Archives of Disease in Childhood, 1972, 47, 344

${ }_{12}$ McIntosh, S, and Aspnes, G T, Pediatrics, 1973, 52, 612.

13 Simone, J V, et al, Cancer, 1975, 36, 770.

${ }^{14}$ Soni, S S, et al, New England fournal of Medicine, 1975, 293, 113.

15 Eiser, C, and Lansdown, R, Archives of Disease in Childhood, 1977, 52, $525 . \frac{\mathrm{\omega}}{\mathrm{T}}$

${ }^{16}$ Coleman, C N, et al, New England fournal of Medicine, 1977, 297, 1249 .

\section{Nature and nurture in child growth}

The interaction of genes and environment affects every aspect of child development. Measurements of height, weight, blood $\mathrm{N}_{\mathrm{N}}$ pressure, and skinfold thicknesses, intelligence, indices of 0 mental stability, and much else have been scrutinised by a whole array of methods to separate the effects of nature and $\overrightarrow{7}$ nurture. Are such analyses helpful?

The answer, like the question, has to be seen in context. When the parental contribution to height, for example, is calculated the answer applies only to the population in which it $\overrightarrow{0}$ was measured. This is true of all characteristics. Heritability is the proportion of the variance of a characteristic determined $\square$ by genetic influences, the remainder of the variance being assumed to be due to environmental factors. Values for heritability of, say, weight will vary with age, sex, race, and other influences. Thus determinants of an individual's weight $\stackrel{\square}{\varnothing}$ may well be quite different when he is an infant, a preschool $\overrightarrow{\overrightarrow{\vec{P}}}$ child, a schoolchild, a teenager, a parent, and an old-age 3 pensioner. Heritability is neither a fixed quantum in an individual nor a biological rule for a species. Furthermore, calculations of heritability are so hedged about with preconditions and confounded by other factors, such as assortative mating, that they may very easily become meaningless except $\frac{0}{3}$ as a statistical exercise. The only reasonable way to calculate heritability would be to examine parents of a given age and $\frac{\delta}{2}$ look at their children when they reach that age; but the environment could hardly be expected to stand still that long.

What researchers do with this muddle in practice is to try $N$ to avoid the major pitfalls and to make the best guesses they $\frac{7}{0}$ can. Thus for blood pressure, which is a variable changing throughout life, they have used twins ${ }^{1}$; they might also use a $N$ method depending on the response to a stimulation rather than absolute levels. For height some have looked only at adult? measurements, ${ }_{3}^{2}$ since these are relatively fixed. For weight, adopted children have been studied, ${ }^{3}$ in an effort to rule out the genetic factors. But however heritability is calculated, do the findings make any practical difference?

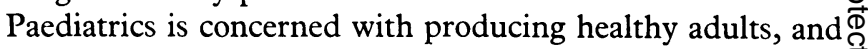
its basic science is the study of growth and development. $\stackrel{\mathbb{Q}}{\Omega}$ Paediatricians are therefore right to seek answers to fundamental questions such as what governs adult weight ando height (and if we stick to height rather than intelligence we응 are spared much emotional discussion-though the arguments and results are remarkably similar). Unless the determinants are known, little can be done to optimise growth. Even so, this is necessarily only half the question. 
The other half concerns trends in individuals. We are discovering that an infant's degree of fatness provides a poor prediction of how fat he will become. ${ }^{4}$ This is not to say that obesity is without hazard in infancy, or that fat children cannot go on to be fat adults. But each child should be treated according to his or her individual characteristics at a given age. Trends or heritabilities of adult characteristics are as irrelevant in an individual case as are, say, the overall statistics for remission and survival to the parents of a child with acute leukaemia. Medicine tries hard to be a science, and rightly so; but in the consulting room it still feels very much like an art.

${ }^{1}$ McIlhany, M L, Shaffer, J W, and Hines, E A, Fohns Hopkins Medical fournal, 1975, 136, 57.

2 Brook, C G D, et al, Annals of Human Biology, 1977, 4, 17.

3 Biron, P, Mongeau, J-G, and Bertrand, D, fournal of Pediatrics, 1977, 91, 555

${ }^{4}$ Poskitt, E M, and Cole, T J, British Medical Fournal, 1977, 1, 7.

\section{Subarachnoid haemorrhage of unknown cause}

The term subarachnoid haemorrhage indicates no more than the presence of blood in the cerebrospinal fluid on lumbar puncture; a more correct term is spontaneous intracranial haemorrhage, which distinguishes the condition from traumatic haemorrhage (a more common cause of blood-stained cerebrospinal fluid) and reminds us that intracranial haemorrhage may occur without the presence of blood in the subarachnoid space.

Faced with the classical presentation of sudden onset of severe headache, vomiting, and neck stiffness, with or without transient loss of consciousness, the physician's task is to assess the likelihood that the cause of the haemorrhage is a remediable lesion and, therefore, the need for intracranial investigations. The choice and extent of the intracranial investigations and the consequent management of the patient are the neurosurgeon's concern, while the later problem of prognosis is left for the physician. Among identifiable causes of subarachnoid haemorrhage are hypertension, aneurysm, angioma, and, less commonly, intracranial tumour (usually malignant), and blood dyscrasias. The prognosis depends in each case on management (which for aneurysm and angioma in turn depends largely on whether the lesion is operable), on the state of the cerebral circulation as indicated by the patient's neurological condition, and on whether or not arterial spasm is shown by angiography.

In some patients investigations fail to identify any causal lesion for the subarachnoid haemorrhage, and until recently the prognosis in such cases has been uncertain. Incomplete follow-up of the patients and the omission of vertebral angiography in most cases have added to the uncertainties. A recent study by Hayward ${ }^{1}$ is therefore welcome in giving some guide to the prognosis. Of 592 patients with subarachnoid haemorrhage admitted during two years, the cause was unknown in 91; of these, 51 had bilateral carotid and vertebral angiography and also computerised tomography (CT scan), so that the presence of a causal lesion had been excluded by every known means (the CT scan has been claimed ${ }^{2}$ to differentiate between primary cerebral haemorrhage and aneurysmal rupture with an accuracy of $90^{\circ}{ }_{6}$ ). The patients' age and sex distribution was similar to that recorded in series of proved cases of intracranial aneurysm, but the incidence of arterial spasm was much lower. There is no conclusive evidence that in cases of this kind haemorrhage is due to small aneurysms that undergo thrombosis or are destroyed at the time of haemorrhage.

None of the 51 patients had a further haemorrhage during the ensuing two months-an observation of practical importance, since further haemorrhage could be expected in that time in half of all patients with a proved aneurysm. Unfortunately, only 41 of the 51 patients had further follow-up, for periods ranging from $3 \frac{1}{2}$ months to $2 \frac{1}{2}$ years; even so, no patient is known to have had a further haemorrhage as yet. Clearly it will be helpful if Hayward's study is continued, and further efforts are made to trace all 51 patients fully investigated. Although such patients can presumably be reassured that there are negligible risks of a further haemorrhage, such a conclusion is unlikely to be acceptable for life insurance, at least on the evidence so far available.

The extent of intracranial investigations in a patient who has had a subarachnoid haemorrhage remains a matter for clinical judgment. If adverse factors such as age, hypertension, cardiovascular disease, and neurological deficit preclude active treatment for a vascular anomaly then angiography is not justified. CT scanning has proved a major advance in this and so many other disorders, ${ }^{3}$ and is particularly valuable in demonstrating the compressing intracranial haematoma that requires urgent evacuation whatever the nature of the causal lesion. The physician must remain aware, however, that even when CT scan appearances suggest an aneurysmal haemorrhage this should not encourage angiography (and particularly vertebral angiography with its hazards) to be performed in the face of adverse clinical factors.

${ }^{1}$ Hayward, R D, Fournal of Neurology, Neurosurgery and Psychiatry, 1977, 40, 926.

' Hayward, R D, and O'Reilly, G V A, Lancet, 1976, 1, 1.

${ }^{3}$ British Medical fournal, 1975, 2, 295.

\section{Television medicine}

Ask a group of doctors what they think of the television coverage of medicine and the one certainty is that they will hold strong views, for doctors, like their patients, find medical programmes compulsive viewing. Many of the documentaries are superb, but the news coverage is less consistent, and occasional programmes provoke a hostile response. This is not due to the outdated attitude that patients are happier if left in ignorance, for most doctors believe that people will take more care of their health if they understand how their bodies function and what can go wrong. Most accept, too, that patients should expect some say in the management of their diseases. The serious criticisms of television medicine concern not its content but its style. They fall into two broad categories: complaints by doctors who have taken part in programmes and allegations by doctor-viewers that programmes too often misinform.

Certainly many doctors who have been interviewed for television programmes have been angered by the final version as screened. Sometimes a 20 -minute filmed discussion is cut down to a minute or two; occasionally nothing appears at all after a half-day given over to the TV team. Our special correspondent (see p 348) found that the regret was caused less by damage to the interviewed doctors' amour propre than by their conviction that their words had been edited-often very selectively-to support the case being made by the television reporter.

For television programmes, whatever their subject matter, 